\title{
Implantless Fixation of a Large Osteocartilaginous Fracture of the Lateral Femoral Condyle in a Child
}

\author{
Jitendra Maheshwari, MS (Orth), Vikram Mhaskar, MS (Orth), MCh (Orth), and \\ Parul Maheshwari Mhaskar, MDS \\ Department of Knee \& Shoulder Surgery, Knee \& Shoulder Clinic, New Delhi, India
}

\begin{abstract}
We describe for the first time a case of lateral femoral condyle fracture following a fall in a 13-year-old child that was reduced arthroscopically using a probe. It was definitively fixed with 0 vicryl sutures in a cruciate pattern after being initially stabilised with 1.5-mm Kirschner wires. Four beath pins carrying sutures were drilled at four opposing quadrants through the reduced fragment into the femur. The sutures were then tied on the lateral cortex of the femur. After fixation, the child was kept non-weight bearing for 6 weeks, partial weight bearing from 6 weeks to 12 weeks and then full weight bearing thereafter. Range of motion (ROM) exercises were commenced on the first postoperative day. At one-year follow-up, the ROM was from $-5^{\circ}$ to $130^{\circ}$, all symptoms disappeared, and complete resumption of all sports activities was allowed.
\end{abstract}

Keywords: Knee, Femur, Fracture, Osteocartilaginous, Fixation, Arthroscopy

Treatment of osteochondral fractures has been traditionally performed via open reduction and internal fixation with various types of hardware. However, minimally invasive procedures that do not necessitate the use of hardware and produce comparable results would be more beneficial to the patient in terms of cosmesis, infection, and stiffness. With this intention in mind, we reviewed the literature on previous techniques that successfully treated similar fractures around the knee and elsewhere in the body to see the feasibility of attempting the same procedure for osteochondral fractures.

Osteochondral fractures are relatively rare in children before calcification of the tidemark as this is important for the stability of the interface between the cartilage and the subchondral bone. However, these fractures can occur in any age group with a peak

Received November 20, 2015; Revised (1st) February 10, 2016;

(2nd) February 29, 2016; Accepted March 21, 2016

Correspondence to: Vikram Mhaskar, MS (Orth), MCh (Orth)

Department of Knee \& Shoulder Surgery, Knee \& Shoulder Clinic, F7

East of Kailash, New Delhi 110065, India

Tel: +91-01126286869, Fax: +91-981-880-6857

E-mail: drvikrammhaskar@gmail.com

This is an Open Access article distributed under the terms of the Creative Commons Attribution Non-Commercial License (http://creativecommons.org/licenses/by-nc/4.0/) which permits unrestricted non-commercial use, distribution, and reproduction in any medium, provided the original work is properly cited. incidence observed in patients around 20 years of age ${ }^{1)}$.

Treatment options vary from excision of the fragment $t^{2)}$ to fixa$\operatorname{tion}^{3,4}$. However, most authors agree that fixation is the most appropriate technique as it restores the native anatomy. Fixation techniques can be performed using bone pegs ${ }^{5,6}$, fibrin adhesive $^{7)}$, absorbable polylactide implants, absorbable pins, screws ${ }^{3,4}$ or sutures ${ }^{8)}$. However, a meta-analysis by Kuhle et al. ${ }^{1)}$ showed that no particular method was superior to the other.

There are potential complications associated with implant loosening and the use of hardware such as lysis caused by biodegradable implants and chondral damage occurring during fixation due to an implant protruding in the joint ${ }^{1-4}$. Suturing can be advantageous in preventing complications associated with implants, allowing early mobilisation, and obtaining excellent fixation. This is especially useful in the paediatric age group because implants can potentially cause cartilage damage and growth plate disturbances and may require an additional surgery for implant removal.

\section{Case Report}

A 13-year-old female child presented with pain, swelling, and inability to bear weight or bend the knee after a fall while running. She had been taken to a hospital immediately after the inju- 
ry and the knee was protected in an extension knee brace. At presentation to our institution, on the first day after the injury, there was a moderate effusion and the range of motion (ROM) was $10^{\circ}-30^{\circ}$. Preoperative X-ray and computer tomography showed a displaced lateral condyle fracture (Fig. 1A). She was scheduled for definitive fixation on the second day after the injury.

\section{Intraoperative Findings and Surgical Technique}

Using a standard anterolateral viewing portal and an anteromedial working portal, a diagnostic arthroscopy was done. The osteochondral fracture of the lateral femoral condyle was diagnosed and reduced using a probe (Fig. 1B). The size of the fragment was $5 \times 5 \mathrm{~cm}$. The fracture was then temporarily fixed with $2-\mathrm{mm}$ Kirschner wires (Fig. 2A).

Four $2.4 \mathrm{~mm}$ beath pins loaded with a 0 vicryl (polyglactin 910; Ethicon, Somerville, NJ, USA) were then passed through the fragment at four quadrants such that the sutures formed a cruciate pattern (Fig. 2B and C). The sutures were delivered and tied on the lateral cortex after making a 2 -cm small incision through the lateral thigh and dissecting down to the bone (Fig. 2D). The fracture site was then probed to assess mobility, and reduction was checked by moving the knee through the full ROM. Fig. 3 shows the knee during surgery performed for fixation of the fracture in a minimally invasive approach.

The operated knee was placed in a long knee brace for two postoperative weeks, and full ROM exercises were commenced on the first postoperative day, but the patient was kept non-weight

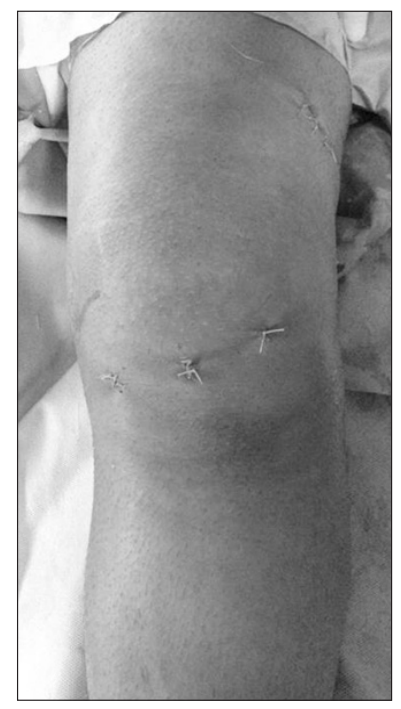

Fig. 3. Minimally invasive fixation of the fracture with three portals.
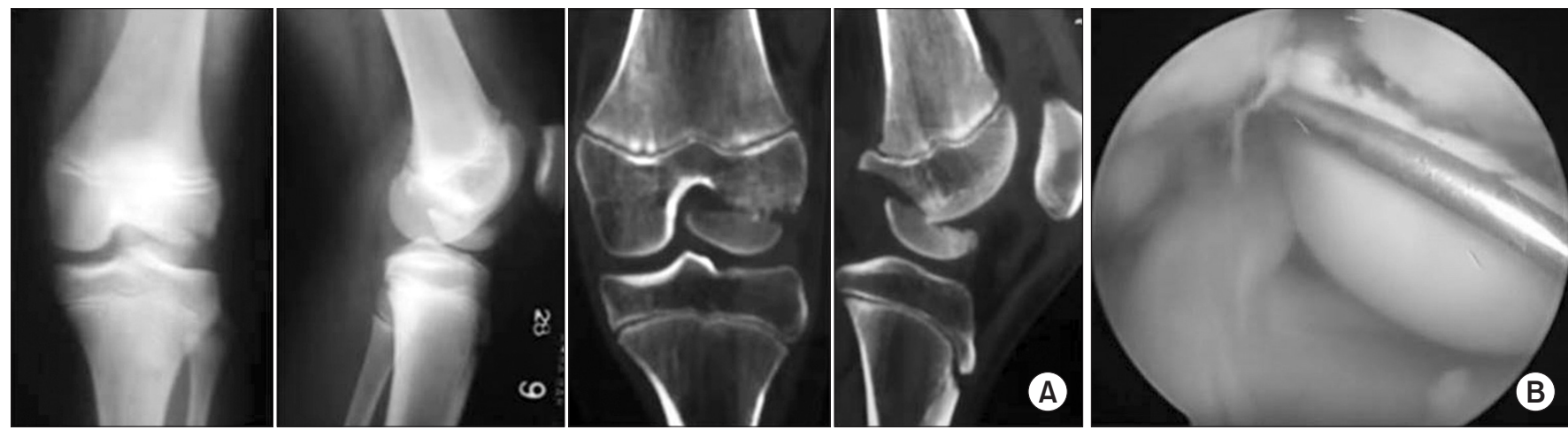

Fig. 1. (A) X-ray and computed tomography scan of fracture. (B) Arthroscopic view of lateral femoral condyle fracture.
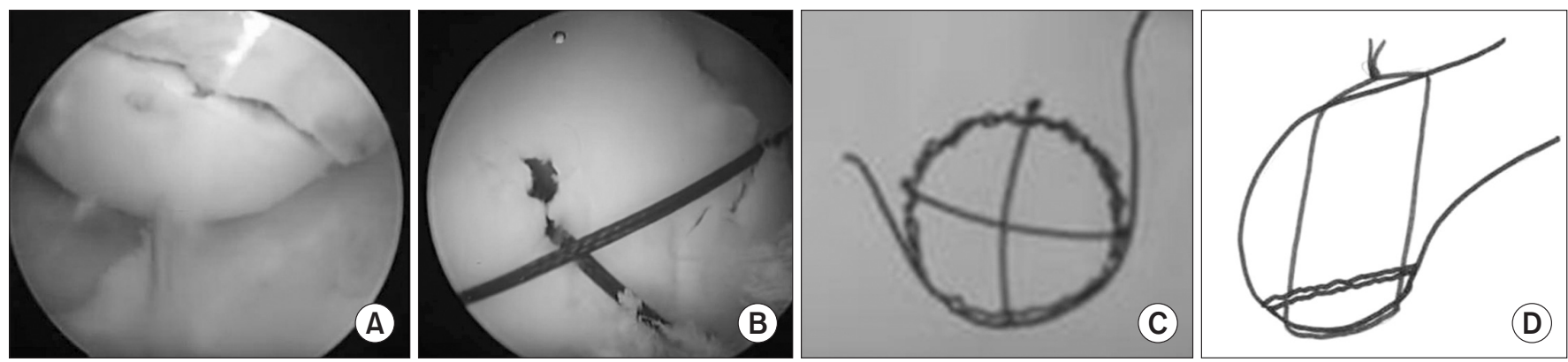

Fig. 2. (A) Temporary fixation with 2 Kirschner wires. (B) Arthroscopic view of cruciate pattern of sutures. (C) Illustration of cruciate pattern. (D) Tying sutures on lateral femoral cortex. 

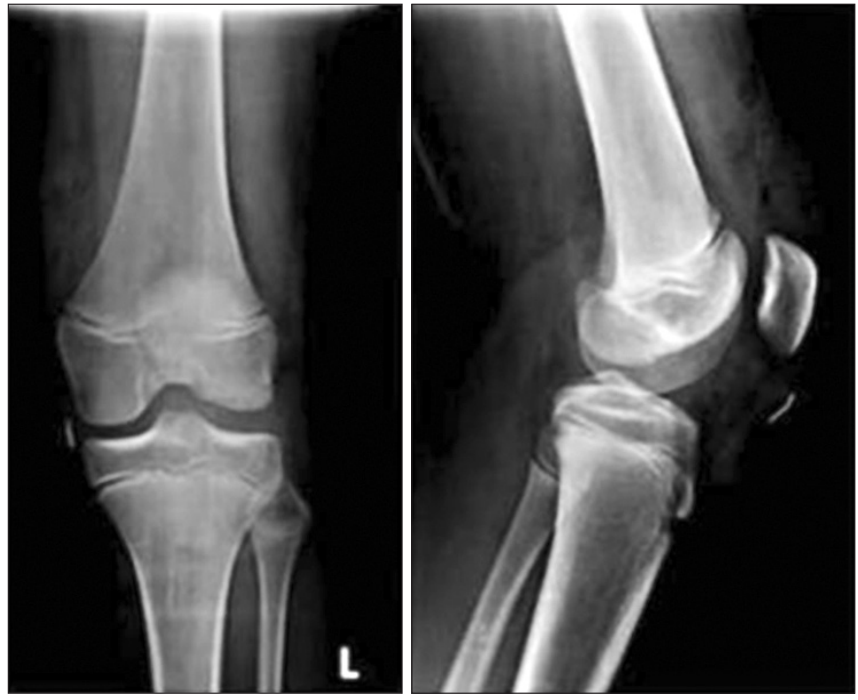

Fig. 4. Postoperative X-ray.

bearing for six postoperative weeks. Partial weight bearing was commenced after six postoperative weeks and full weight bearing at three postoperative months. Postoperative X-ray showed good reduction of the fragment with restoration of the joint line (Fig. 4). She achieved full ROM ( $5^{\circ}$ of hyperextension to $130^{\circ}$ of flexion) within three postoperative months, excellent quadriceps strength, and a normal gait without any symptoms. She returned to full sports activities at six postoperative months and continued to play basketball at club level at two year of follow-up. The International Knee Documentation Committee score was 95.65.

\section{Discussion}

The evolution of fixation of osteochondral fractures has moved from excision and fixation through open surgery with metallic implants to the use of bioabsorbable implants and less invasive methods such as arthroscopy. Recent literature has suggested using fibrin glue and even sutures for fixation of such fractures ${ }^{8,9)}$. However the occurrence of such fractures in the pediatric age group is relatively rare. In our case, the patient was a 13-year-old child, and our concerns were accurate reduction, stability of fracture reduction, early mobilisation, and cosmesis.

For management of the pediatric fracture, we also had to consider growth plate disturbances and future surgery for hardware removal that may impede growth. Thus, it was important to use a fixation device that neither potentially affects the growth plate nor requires future removal while providing stable fixation. Fractures in adults take longer to heal than those in the pediatric age group, and thus have the potential to displace if not rigidly fixed.
We reported the first case of suture bridge fixation for a large osteochondral fracture of the lateral femoral condyle of the femur in a child. This technique was initially applied to capitellum fractures ${ }^{10)}$. Previous fixation methods including suture bridge fixation have shown equivocal results in the adult age group. However, the suture bridge technique has the additional advantage of being an arthroscopic technique.

In comparison to the technique described by Bowers and Huffman $^{8}$, our technique was performed in an acute setting in a pediatric patient. The presence of raw opposing surfaces did not require drilling through the subchondral bone to improve vascularity. The fragment in this case was much larger $(5 \times 5 \mathrm{~cm})$ and not lying free in the joint. Thus, reduction required only manipulation of the fragment with a probe to ensure anatomical position.

Although the postoperative rehabilitation protocol was the same as that in previous studies involving ROM exercises and quadriceps strengthening exercises immediately after surgery, we permitted weight bearing at six postoperative weeks.

The potential limitations of this technique include the potential loss of reduction due to inadequate tying of the sutures and the steep learning curve.

\section{Conflict of Interest}

No potential conflict of interest relevant to this article was reported.

\section{References}

1. Kuhle J, Angele P, Balcarek P, Eichinger M, Feucht M, Haasper C, Alexander G, Jung T, Lill H, Marquass B, Osti M, Rosenberger R, Salzmann G, Steinwachs M, Voigt C, Vogt S, Zeichen J, Niemeyer P. Treatment of osteochondral fractures of the knee: a meta-analysis of available scientific evidence. Int Orthop. 2013;37:2385-94.

2. Rosenberg NJ. Osteochondral fractures of the lateral femoral condyle. J Bone Joint Surg Am. 1964;46:1013-26.

3. Walsh SJ, Boyle MJ, Morganti V. Large osteochondral fractures of the lateral femoral condyle in the adolescent: outcome of bioabsorbable pin fixation. J Bone Joint Surg Am. 2008;90:1473-8.

4. Wachowski MM, Floerkemeier T, Balcarek P, Walde TA, Schuttrumpf JP, Frosch S, Dathe H, Kertesz A, Sturmer KM, Frosch KH. Mid-term clinical and MRI results after refixation of osteochondral fractures with resorbable implants. Z 
Orthop Unfall. 2011;149:61-7.

5. Ahstrom JP Jr. Osteochondral fracture in the knee joint associated with hypermobility and dislocation of the patella: report of eighteen cases. J Bone Joint Surg Am. 1965;47:1491502.

6. Lindholm S, Pylkkanen P, Osterman K. Fixation of osteochondral fragments in the knee joint: a clinical survey. Clin Orthop Relat Res. 1977;(126):256-60.

7. ten Thije JH, Frima AJ. Patellar dislocation and osteochondral fractures. Neth J Surg. 1986;38:150-4.
8. Bowers AL, Huffman GR. Suture bridge fixation of a femoral condyle traumatic osteochondral defect. Clin Orthop Relat Res. 2008;466:2276-81.

9. Gul R, Khan F, Maher Y, O'Farrell D. Osteochondral fractures in the knee treated with butyl-2-cyanoacrylate glue: a case report. Acta Orthop Belg. 2006;72:641-3.

10. Sodl JF, Ricchetti ET, Huffman GR. Acute osteochondral shear fracture of the capitellum in a twelve-year-old patient: a case report. J Bone Joint Surg Am. 2008;90:629-33. 